\title{
Eureka: um programa de mentoria de alunos de Medicina com engajamento e alta adesão
}

\author{
Eureka: a mentoring program for medical students with high levels of commitment and attendance \\ Luciana Antunes de Almeida Secchi' ${ }^{1}$ (D) |lusecchi@terra.com.br \\ Bruno Alba Vieira1 (D) alba.br@gmail.com
}

\begin{abstract}
RESUMO
Introdução: A mentoria vem sendo utilizada como instrumento para o desenvolvimento profissional e pessoal de estudantes de medicina. Relatamos a evolução do grupo de mentoria de alunos de graduação em medicina da Universidade Federal da Grande Dourados (UFGD), que perdura por longo tempo, com excelente aderência e amplo aproveitamento, e satisfação dos participantes com o processo.

Relato de experiência: O programa de mentoria de alunos de medicina da UFGD se iniciou no quinto ano de existência do curso, encontrandose em contínua atividade por mais de 15 anos. Há três anos, tornou-se projeto de extensão universitária. São realizadas discussões semanais sobre assuntos práticos, morais e éticos relacionados à profissão médica. Os participantes são mentorados por tempo variável, eventualmente até alguns anos após a graduação. A atividade é voluntária para mentora e mentorados. Muito raramente ocorrem faltas aos encontros, e semanalmente os participantes escrevem sobre suas reflexões advindas das sessões de mentoria, recebendo respostas individuais. Também assumem as responsabilidades pela realização operacional das reuniões e pela indicação de novos participantes. Sessões adicionais de mentoria individual são frequentes.
\end{abstract}

Discussão: Programas de mentoria pequenos ou informais, se bem executados, podem ser bem-sucedidos, obtendo resultados positivos significativos, sem custo para as instituições. Os alunos que participam do programa Eureka sentem-se acolhidos e atendidos em suas necessidades de orientação, e se mostram comprometidos e satisfeitos com o processo. Não observamos nesse grupo problemas de assiduidade, de engajamento, nem de falta de feedback.

Conclusão: O programa Eureka, com regras de funcionamento claras e constantes, mentoria adequada e alunos bem selecionados, constitui eficiente ferramenta em nossa universidade, contribuindo, assim, para a formação de estudantes e médicos mais motivados, preparados, realizados e úteis à sociedade.

Palavras-chave: tutoria; mentores; feedback formativo; estudantes de medicina; educação médica.

\section{ABSTRACT}

Introduction: Mentorship has been used as an instrument for professional and personal development of medical students. This article reports on the development of the medical student mentorship program at the Federal University of Grande Dourados (UFGD), which has been active for many years with excellent adherence and high levels of satisfaction and progress of the participants.

Experience report: The UFGD medical student mentorship program began in the fifth year of the medical course's existence and has been continuously active for over 15 years. Three years ago, it became a university extension project. Weekly discussions regarding practical, moral and ethical aspects of the medical profession take place. The participants are mentored for varying periods of time, possibly up to a few years after undergraduate training. The activity is voluntary for both mentor and mentees. Absences from the meetings are very rare and the mentees write a weekly log about their reflections from the mentoring sessions, to which the mentor replies individually. They also take on the responsibilities of organizing the meetings and of indicating new participants. Additional individual mentoring sessions are frequent.

Discussion: Small or informal mentorship programs, if well executed, can be successful, obtaining positive results at no cost to the institution. The students who take part in the Eureka program feel welcome, guided and show commitment and satisfaction with the process. We have not observed in this group any issues with engagement and attendance, or lack of feedback.

Conclusion: The Eureka program, with its clear and constant rules, adequate mentoring and well selected students, constitutes an efficient tool in our university, therefore, contributing to the education of more motivated, prepared and self-fulfilled students and physicians.

Keywords: mentoring; mentors; formative feedback; students, medical; education, medical.

\footnotetext{
${ }^{1}$ Universidade Federal da Grande Dourados, Dourados, Mato Grosso do Sul, Brasil.
}

Editora: Lia Marcia Cruz da Silveira.

Recebido em 25/02/21; Aceito em 26/04/21.

Avaliado pelo processo de double blind review. 


\section{INTRODUÇÃO}

O estresse percebido pelos estudantes de medicina, resultante da alta carga horária de atividades, do pouco tempo para o lazer e do ambiente de alta competitividade', e as características do curso, como corpo de professores heterogêneo e turmas numerosas, não favorecem o ensino individualizado e a construção compartilhada de conhecimentos. Tampouco favorecem o desenvolvimento de um relacionamento entre docentes e discentes que sirva como suporte aos alunos no trânsito pelas etapas profissionais e pessoais que devem atravessar durante sua evolução de estudante para médico em início de carreira.

Mentoria pode ser definida como um processo em que alguém de reconhecida experiência, bom conceito pessoal e profissional e perfil empático (mentor) guia um indivíduo mais jovem (mentorado) em seu crescimento profissional, levando-o a reexaminar e reinterpretar suas próprias ideias, aprendizado e escolhas ${ }^{2}$. A mentoria vem sendo amplamente e há longo tempo utilizada em vários países como instrumento para o desenvolvimento profissional e pessoal de estudantes de medicina e médicos ${ }^{2,3}$.

Quando se trata de alunos da área da saúde, a mentoria se constitui num componente crítico para o sucesso acadêmico ${ }^{4}$. Bons resultados dependem grandemente das características dos mentores e de sua condução apropriada da mentoria ${ }^{2,4}$.

Na mentoria de alunos de graduação, a abordagem consistente, embora, por várias razões, difícil de se obter, é importante para que se atinjam plenamente os objetivos do processo $^{5}$. Descrições da experiência de grupos de mentoria em escolas médicas brasileiras relatam baixa frequência dos alunos aos encontros em grupo e pouco envolvimento no processo de mentoria $^{6,7}$.

Relatamos a evolução do grupo de mentoria de alunos de graduação em medicina da Universidade Federal da Grande Dourados (UFGD), em Dourados, Mato Grosso do Sul, Brasil, ressaltando as características consideradas relevantes para a manutenção do grupo por longo tempo, com aderência praticamente total dos alunos participantes e amplo aproveitamento e satisfação de mentorados e mentora com o processo de mentoria nos moldes utilizados.

\section{RELATO DE EXPERIÊNCIA}

\section{Surgimento do programa}

O curso de medicina da UFGD foi criado em 1999, com o início das aulas da primeira turma em 8 de maio de 2000. O Hospital Universitário da UFGD constitui centro de referência em atendimento à saúde para os 33 municípios da macrorregião de Dourados, no estado de Mato Grosso do Sul, atendendo uma população de aproximadamente 820 mil habitantes ${ }^{8}$.
Atualmente são admitidos 80 alunos por ano, divididos em duas turmas que iniciam suas atividades estudantis no primeiro e segundo semestres letivos de cada ano.

O programa de mentoria de alunos do curso de medicina da Faculdade de Ciências da Saúde da UFGD iniciou suas atividades no ano de 2004, com discussões sobre assuntos práticos, morais e éticos relacionados à profissão médica. Por um breve período, as conversas eram espontâneas e informais, ao final das aulas teóricas e/ou em ambulatórios sob a responsabilidade da mentora, tendo rapidamente evoluído para discussões que passaram a ser programadas e estruturadas. As sessões eram oferecidas aos alunos matriculados no quarto ano do curso, por adesão voluntária, conduzidas por uma única mentora, médica especialista em área clínica e docente da universidade. O número de alunos interessados na atividade foi aumentando de maneira gradual, e, também espontaneamente, foram sendo agregados alunos de outros períodos do curso.

\section{Evolução}

Com o aumento do número de alunos no grupo e o interesse demonstrado pelas atividades, estabeleceu-se que os encontros ocorreriam em horário noturno, fora da grade de aulas curriculares. $O$ grupo definiu-se em seu objetivo com a finalidade de "ensinar coisas que não se podem encontrar no Google", frase utilizada como identificador secundário e descritor do grupo até os dias de hoje. O programa de mentoria foi nomeado "Eureka" pelos próprios alunos, por entenderem que as discussões os levavam à descoberta de si mesmos, de seus pontos fortes ou vulneráveis, a respostas a questionamentos e a escolhas conscientes, as quais, antes da mentoria, Ihes pareciam ocultas ou inacessíveis. Depois de quase dez anos de atividades regulares, com discentes integrando-se ao grupo espontaneamente, decidiu-se manter no grupo de mentoria apenas alunos matriculados a partir do terceiro ano do curso médico. Há cerca de três anos foi necessário restringir o número de participantes para favorecer o método que consideramos mais proveitoso e satisfatório para mentorados e mentora.

Em 2018, depois de 14 anos do início da atividade, o grupo de mentoria foi oficializado como um projeto de extensão universitária, cuja renovação documental no momento encontra-se prejudicada pela paralisação temporária de algumas atividades administrativas da universidade, imposta pela pandemia de covid-19.

\section{Características do grupo de mentoria de alunos de medicina da UFGD}

Até o início da pandemia de covid-19, as reuniões ocorriam presencialmente, em sala de reuniões - não em sala de aulas do tipo auditório. A disposição dos participantes ao 
redor de uma mesa favorece a integração e proximidade, e as características reflexivas das discussões. Os encontros eram realizados, durante o ano todo, a cada três semanas, interrompendo-se a frequência apenas ao final do ano para o período de férias de dezembro a início de janeiro. A duração de cada encontro era de duas a três horas, com variação natural conforme a evolução das discussões e interações.

Com a interrupção do ano letivo pelas restrições impostas pela pandemia de covid-19, as atividades de mentoria foram interrompidas por dois meses e, então, retomadas, a pedido dos próprios alunos, no formato de reunião remota em plataforma virtual, com a duração de duas horas ou pouco mais cada encontro. São de responsabilidade dos alunos a convocação do grupo para os encontros, a transmissão de documentos para discussão e recados, e a geração do link para acesso ao ambiente de reunião virtual. Um dos alunos do grupo permanece como auxiliar da mentora para operacionalizar as tarefas de organização.

Os participantes recebem o link por meio de grupo em rede social na véspera da reunião e ingressam no ambiente virtual alguns minutos antes do horário agendado para início. As reuniões se iniciam pontualmente, mantendo-se, em geral, o dia da semana e o horário. Reuniões adicionais em outros horários podem ser agendadas, em acordo entre mentora e mentorados, conforme a necessidade e conveniência do grupo. Durante a sessão de mentoria, todos os participantes mantêm, obrigatoriamente, as câmeras ligadas e postam-se diretamente de frente para a câmera, visando a favorecer a interação pessoal e o ambiente amigável na sala virtual.

Na retomada das reuniões regulares após a interrupção em março e abril de 2020, as reuniões no formato virtual ocorreram durante um mês em periodicidade quinzenal. $\mathrm{Na}$ sequência, passaram a ser realizadas semanalmente, sem interrupção, por solicitação dos alunos mentorados. Os alunos relataram ser a atividade importante para se manterem ligados à universidade e se sentirem mais produtivos e animados, enquanto as aulas não retornam à frequência habitual, mencionando os encontros do grupo de mentoria como um fator positivo na manutenção de sua saúde mental durante um período de perdas e restrições.

A temática é definida pela mentora, contemplando aspectos relacionados à conduta profissional dos alunos como estudantes de medicina e futuros médicos (quadro 1). Aos mentorados é permitido sugerir temas, os quais podem ser contemplados em discussões futuras, quando oportuno.

Os temas são introduzidos em sequência flexível, conforme a evolução das discussões, porém buscando corresponder ao contexto comunitário e social e às necessidades prementes do grupo em cada momento, e favorecer uma sequência didática para reinterpretação de assuntos e construção de conhecimento, opiniões e escolhas. Existe compromisso de sigilo quanto ao que se discute nas reuniões do grupo, por questões éticas e para que os participantes se sintam seguros para expor livremente seus relatos e opiniões.

A abordagem dos temas é dinâmica, a depender da resposta, do interesse e do aproveitamento dos mentorados. Ocorre no formato de roda de conversa coordenada pela mentora, e eventualmente se utilizam métodos audiovisuais (independentemente de reunião presencial ou remota). Eventualmente, aulas sobre assuntos relacionados proferidas por outros profissionais servem de base para a discussão de assuntos necessários ao desenvolvimento dos mentorados. Há reuniões que ocorrem com a participação de outras pessoas, médicos ou não, que comparecem como colaboradores em assuntos pertinentes, assim como de egressos do grupo de mentoria, que retornam para colaborar com o relato de suas experiências, aptidões e dificuldades no início da carreira.

A presença nas reuniões só é permitida aos alunos regularmente integrados ao grupo de mentoria e a colaboradores eventuais. O tempo de permanência de cada aluno no grupo é definido pela mentora, considerando-se o aproveitamento e a necessidade de amadurecimento do aluno no processo, a adaptação ao grupo, a participação ativa nas discussões e o cumprimento dos compromissos de responsabilidade, pontualidade e assiduidade. A possibilidade de faltas é limitada e essas devem ser sempre justificadas.

Quadro 1. Temas abordados nas reuniões de mentoria

Reflexões sobre o médico que se quer ser

Reflexões críticas sobre o médico que não se quer ser

Especialidades médicas

Controle do estresse

Planejamento de carreira

Ensino médico e educação continuada

Métodos de estudo eficientes

A morte e outras situações difíceis no quotidiano médico

Planejamento financeiro

Ética médica

Mercado de trabalho

Medicina baseada em evidências

Discussões sobre situações práticas vividas pelos mentorados ou por pessoas próximas a eles

Equilíbrio da vida pessoal com a vida profissional Conduta profissional como acadêmicos de medicina Saúde física e mental 
Depois de cada reunião, os mentorados devem enviar à mentora, por e-mail, um texto contendo reflexões sobre o significado pessoal do assunto discutido, chamado de feedback. O feedback pode ser enviado até um dia e meio após a reunião, é sigiloso e sempre respondido individualmente em até 24 horas pela mentora. Isso é feito com o intuito de auxiliar na interpretação das reflexões realizadas pelo mentorado, sanar dúvidas ou atender a situações pessoais que o aluno prefira não expor perante o grupo. As impressões, dúvidas e necessidades dos mentorados, expressas nos textos de feedback, são usadas pela mentora para monitoramento dos resultados das atividades realizadas, além da evolução e das necessidades do grupo e dos alunos individualmente, e para programação de conteúdo das reuniões subsequentes.

Reuniões de mentoria individual são frequentemente realizadas para análises mais particulares de assuntos de interesse pessoal dos mentorados, observações ou orientações personalizadas, a depender da necessidade percebida pela mentora ou pelo mentorado. As sessões de mentoria individual ocorrem em horários diversos das reuniões habituais do grupo.

\section{Composição do grupo}

As regras para integrar o grupo de mentoria foram estruturadas em um documento redigido pelos próprios alunos, com base no programa de mentoria de alunos de medicina da Virginia Commonwealth University ${ }^{9}$, e revisado pela mentora. Tal documento funciona como um contrato de adesão, apresentado pelos mentorados aos colegas postulantes a vagas no grupo de mentoria, os quais são integrados ao grupo apenas se concordantes com os termos. A participação é voluntária e isenta de custos financeiros.

Os alunos do curso de medicina da UFGD podem se apresentar como postulantes a uma vaga no grupo de mentoria se indicados por colegas já participantes, após análise e concordância com o regulamento. Passam por rápida entrevista com a mentora (em que se busca avaliar a capacidade de comprometimento e disposição do aluno para o trabalho em grupo). Devem ainda responder, por escrito, a duas perguntas: "O que espera encontrar no grupo de mentoria?" e "Em que acha que sua participação no grupo de mentoria pode beneficiá-lo como pessoa e como futuro médico?". Os postulantes às vagas, se considerados aptos a ingressar no programa, vão sendo integrados progressivamente, conforme a disponibilidade de vagas.

Estabeleceu-se como limite, em razão de haver apenas uma mentora, o número máximo de 15 alunos participantes regulares, do quinto semestre do curso ou acima. Novos participantes só podem ser admitidos quando alguém se desliga do grupo por decisão da mentora ou escolha própria.
A participação é suspensa por iniciativa da mentora quando o participante não cumpre com os compromissos assumidos com o grupo, não se adapta ou quando se considera que já teve bom proveito e evolução com sua participação e esse ciclo se concluiu - neste caso, param de frequentar o grupo, mas permanecem mentorados individualmente, se o desejarem. Vários egressos da mentoria continuam em contato próximo e frequente com a mentora para discussões individuais por até três ou quatro anos após a conclusão do curso de medicina. Mesmo contando com apenas uma mentora, já passaram pelo grupo de mentoria de alunos de medicina da UFGD como mentorados mais de duas centenas de discentes.

\section{DISCUSSÃO}

Programas de mentoria informais existem, e estima-se que sejam frequentes ${ }^{10}$. Se bem executados, podem ser bemsucedidos, obtendo-se resultados positivos significativos sem custos financeiros ou operacionais para as instituições ${ }^{2,10}$. Apesar de a atividade de mentoria de alunos de medicina na UFGD ter se iniciado de modo informal e despretensioso, contando com apenas uma mentora, o programa já perdura por mais de 15 anos, em funcionamento regular. Os alunos que participam do programa sentem-se acolhidos e bem atendidos em suas necessidades de orientação, desfrutam de liberdade de acesso e disponibilidade da mentora, além de se mostrarem engajados e entusiasmados com o processo, de acordo com o que repetidamente relatam em seus textos de feedback.

Boa parte dos egressos desse programa, agora médicos, se manifesta satisfeita com a evolução pessoal e profissional auto-observada em razão da mentoria. Relatam que, por terem sido "preparados" durante o processo, sentem-se mais adequados em relação a suas escolhas pessoais e profissionais, condutas e posicionamentos em relação às diversas situações delicadas, de pressão ou mesmo agressão com que se defrontam na continuidade de sua formação ou no exercício da profissão. Muitos egressos, hoje médicos residentes ou já plenamente inseridos no mercado de trabalho, mantêm contato com a mentora por vontade própria ou quando se encontram em situações em que sentem necessidade de apoio e orientação e para compartilhar conquistas ou alegrias. Alguns retornam ao grupo como colaboradores ou para relatos e depoimentos aos mais jovens.

A adesão dos participantes é de quase 100\% de presença aos encontros, tanto quando estes eram possíveis presencialmente quanto ocorrendo por via remota em razão da pandemia de covid-19. Faltas apenas ocorrem muito raramente, por motivo de força maior (acidentes ou perdas familiares). Nas reuniões remotas semanais, nos últimos dez meses, ocorreram apenas quatro faltas individuais de alunos aos encontros, todas 
justificadas. Os textos de feedback são enviados regularmente e no prazo solicitado. Quando raramente dispensados de obrigatoriedade pela mentora, cerca de metade dos alunos, ainda assim, os envia espontaneamente. Infere-se que compreendem a importância desse exercício na sua evolução no processo de mentoria e que o compromisso com o procedimento foi totalmente adotado por eles, diferentemente do que vemos relatado em outros programas de mentoria em universidades brasileiras $s^{6,11,12}$.

Do ponto de vista da mentora, a atividade de mentoria de alunos de medicina da UFGD motivou o aprimoramento de habilidades específicas de mentoria e de ensino, melhor conhecimento do corpo discente da universidade e dos problemas da instituição. Obtêm-se satisfação pessoal e troca profissional e afetiva com os mentorados, os egressos do programa e os diversos colaboradores que generosamente prestam importante contribuição para o grupo. Observamos em nossa atividade os benefícios relatados por programas de mentoria diversos no nosso e em outros países ${ }^{2,4,7,13}$.

\section{CONCLUSÃO}

O programa de mentoria de alunos do curso de medicina da UFGD vem sendo considerado bem-sucedido pela mentora e pelos alunos participantes, que o expressam direta e objetivamente a cada semana em suas falas durante as reuniões, em seus textos de feedback, além de o demonstrarem pela assiduidade, pelo apego ao grupo, pelo entusiasmo nas atividades e pelo apreço e zelo por sua vaga num programa com vagas limitadas.

Consideramos uma limitação a escassez de profissionais/ docentes com o perfil adequado para que possam ser mentores, dispostos a conceder seu tempo para a atividade e dedicar-se aos alunos com benefícios também para si mesmos. Mentores em maior número possibilitariam atender mais alunos, porém é nossa opinião que nem todo profissional ou docente pode ser um bom mentor. É necessário que os mentores sejam detentores de certos atributos pessoais e profissionais para o adequado e proveitoso andamento da atividade - o mentor deve ser reconhecido como um bom professor, modelo profissional, fonte de informação, conselheiro, incentivador e defensor de seus mentorados, trabalhando com entusiasmo para orientá-los e incentivá-los durante sua educação e treinamento ${ }^{4}$. Também precisa ter interesse genuíno no desenvolvimento de seus protegidos, e acreditamos que não deve ser compelido a mentorar alunos em quem não encontre estímulo e afinidade e com quem não se sinta à vontade para partilhar suas experiências profissionais e pessoais.

Do mesmo modo, nem todos os alunos desejam programas de mentoria ou se beneficiam deles. Assim sendo, mantemos a opção e a opinião em favor da mentoria absolutamente voluntária para os alunos e também para os mentores, de modo a preservar genuínos os objetivos, por definição, de um programa de mentoria.

Entendemos que a mentoria deve ser um relacionamento de longo prazo entre o mentor e o mentorado, não devendo se restringir ao período limitado de uma disciplina curricular. Também em nossa opinião, o mentor deve ter em plena consciência facilitar o progresso pessoal e profissional do mentorado, no sentido de guiá-lo para que chegue às suas próprias conclusões e escolhas de forma produtiva e consciente, e deve saber que esse contato humano intenso dificilmente pode ser remunerado financeiramente.

Maiores interesse, envolvimento e incentivo da universidade e da coordenação dos cursos também seriam desejáveis. Egressos dos programas de mentoria costumam demonstrar comportamento mais ético, equilibrado e adequado, além de o fato de ser mentorado ter importante influência no desenvolvimento pessoal, no planejamento e na escolha da carreira ${ }^{13}$, o que é muito desejável na formação dos futuros médicos, beneficiando a instituição de ensino e a sociedade como um todo.

O exercício da redação do feedback depois de cada atividade da mentoria, a análise dos textos e a resposta individualizada pela mentora têm se demonstrado fundamentais para o aproveitamento das discussões e sua conversão de reflexões a mudanças de comportamento e de reação para padrões mais favoráveis. Em nossa prática, depois que esse exercício foi adotado como rotina, ele vem sendo importante acelerador do aproveitamento dos alunos nas atividades do programa.

Temos obtido bons resultados no programa de mentoria Eureka para alunos de medicina da UFGD, superando dificuldades comumente relatadas em outras experiências em nosso país ${ }^{6,12,14}$, em especial no que se relaciona à assiduidade dos alunos mentorados e à manutenção do engajamento e compromisso com o processo por parte dos mentorados e da mentora. Com base em nossa experiência, programas bem estruturados de mentoria de alunos de graduação em medicina, com regras claras e funcionamento constante, mentor(es) e alunos com perfil adequado, participantes bem selecionados e cumpridores de seus papéis, independentemente do tamanho e de serem formais ou informais na estrutura das universidades, podem constituir excelente e eficiente ferramenta na formação de estudantes e médicos mais motivados, preparados, conscientes, realizados e úteis à sociedade. A consideração dos pontos citados neste relato pode contribuir para o sucesso de futuros programas de mentoria a serem iniciados, assim como para o aprimoramento de programas já existentes. 


\section{CONTRIBUIÇÃO DOS AUTORES}

Luciana Antunes de Almeida Secchi: conceptualização e escrita do rascunho original.

Bruno Alba Vieira: contribuição na escrita, análise e edição do relato.

\section{CONFLITO DE INTERESSES}

Declaramos não haver conflito de interesses.

\section{FINANCIAMENTO}

Declaramos não haver financiamento.

\section{REFERÊNCIAS}

1. Bergmann C, Muth T, Loerbroks A. Medical students' perceptions of stress due to academic studies and its interrelationships with other domains of life: a qualitative study. Med Educ Online. 2019;24(1).

2. Taherian K, Shekarchian M. Mentoring for doctors. Do its benefits outweigh its disadvantages? Med Teach. 2008 Jan 3;30(4):e95-9. doi: 10.1080/01421590801929968.

3. Woessner R, Honold M, Stehle I, Stehr S, Steudel WI. Faculty mentoring programme: ways of reducing anonymity. Med Educ. 1998;32(4):441-3.

4. Cho CS, Ramanan RA, Feldman MD. Defining the ideal qualities of mentorship: a qualitative analysis of the characteristics of outstanding mentors. Am J Med. 2011;124(5):453-8.

5. Tan YS, Teo SWA, Pei Y, Sng JH, Yap HW, Toh YP, et al. A framework for mentoring of medical students: thematic analysis of mentoring programmes between 2000 and 2015. Adv Heal Sci Educ. 2018;23(4):67197. doi: 10.1007/s10459-018-9821-6.
6. Moreira SNT, de Albuquerque ICS, Pinto Junior FEL, Gomes AHB. Programa de Mentoria do curso de Medicina da Universidade Federal do Rio Grande do Norte: atividades integrativas em foco. Rev Bras Educ Med. 2020;44(4):e169.

7. Bellodi PL. Mentors, students, and the undergraduate medical course: a virtuous circle. Rev Bras Educ Med. 2011;35(3):382-8.

8. Gonçalves E, Lima H, Gonçalves J, Attilio J, Leite M. Carta de serviços ao usuário do Hospital Universitário da UFGD. Dourados: Hospital Universitário da UFGD, Empresa Brasileira de Serviços Hospitalares, Ministério da Educação; 2018 [acesso em 22 fev 2021]. Disponível em: https://www.gov.br/ebserh/pt-br/hospitais-universitarios/regiaocentro-oeste/hu-ufgd/acesso-a-informacao/programas-e-projetos/ AnexoResoluo054CartadeServios.pdf.

9. Swanson KE. Mentorship manual for medical students. Virginia: School of Medicine, Medical College of Virginia Campus, Virginia Commonwealth University; 2001 [acesso em 22 fev 2021]. Disponível em: https://medschool. vcu.edu/media/medschool/documents/MentorshipManual.pdf.

10. Bligh J. Mentoring: an invisible support network. Med Educ. 1999;33(1):2-3.

11. Bellodi PL, Chebabo R, Abensur SI, Martins M de A. Mentoring: ir ou não ir, eis a questão: um estudo qualitativo. Rev Bras Educ Med. 2011;35(2):237-45.

12. Martins AF, Bellodi PL. Mentoring: uma vivência de humanização e desenvolvimento no curso médico. Interface Comun Saúde Educ. 2016;20(58):715-26.

13. Burgess A, van Diggele $C$, Mellis C. Mentorship in the health professions: $a$ review. Clin Teach. 2018;15(3):197-202.

14. Gonçalves MCN, Bellodi PL. Mentores também precisam de suporte: um estudo sobre suas dificuldades e recursos na escola médica. Sao Paulo Med J. 2012;130(4):252-8. 